\title{
Symmetries and the identity of physical states
}

\author{
Simon Friederich \\ s.m.friederich@rug.nl \\ University of Groningen, University College Groningen, Hoendiepskade \\ 23/24, NL-9718 BG Groningen and \\ University of Groningen, Faculty of Philosophy, Oude Boteringestraat 52, \\ NL-9712 GL Groningen, The Netherlands
}

\begin{abstract}
The paper proposes a combined account of identity for physical states and direct empirical significance for symmetries according to which symmetry-related state variables designate distinct physical states if and only if the symmetry that relates them has direct empirical significance. Strengthening an earlier result, I show that, given this combined account, the local gauge symmetries in our leading contemporary theories of particle physics do not have any direct empirical significance.
\end{abstract}

Keywords: Symmetries, Identity, Empirical significance, Gauge theories

\section{Introduction: Identifying physical states}

Understanding a physical theory involves understanding the ways the world can be if the theory is true. One important aspect of the latter is having criteria of identity for its physical states.

Individuating a theory's distinct physical states comprises, first, the broad subtask of clarifying in which way competent scientists use the formalism of the theory and employ it to predict and/or explain certain empirical phenomena. Second, it comprises the subtask of specifying those variables of the formalism that have the semantic and/or linguistic role of actually designating the world's physically possible distinct states. One may refer to these variables as the ontic variables of the theory, and for some theories identifying them can be a real challenge. In quantum theory, for example, it is notoriously controversial whether quantum states are (among the) ontic variables of the theory or whether they are rather-in some sense to be clarified- "epistemic", i.e. more akin to classical probability distributions over whatever one regards as the true physical states that underlie quantum theory. ${ }^{1}$

In this paper, I assume that, for some theory of interest, these first two subtasks have already been solved. This may still leave open a third subtask that will stand in the focus of this paper: namely, to clarify how ontic variables are mapped onto the physical states, i.e. whether distinct ontic variables sometimes designate one and

\footnotetext{
${ }^{1}$ See (Leifer 2014) for a highly useful review of, on the one hand, the motives to doubt that quantum states are ontic and, on the other hand, the difficulties that arise from assuming that they are not ontic.
} 
the same physical state or, put more generally, how one can individuate a theory's physical states by starting from the ontic variables. To be able to sidestep the question of whether quantum states are ontic or epistemic, I will focus on classical theories.

\section{Physical states and symmetries}

A physical theory's symmetry transformations ("symmetries") connect ontic variables that designate empirically indistinguishable physical states. A possible reason of why states that are designated by distinct ontic variables can be empirically indistinguishable is that they are not physically distinct at all, i.e. distinct ontic variables which apply to a system in empirically indistinguishable situations may designate identical physical states. Accordingly, a promising approach to identifying a theory's distinct physical states is by focusing on its symmetries, notably by identifying and classifying them. Fortunately, there exists a well-established strategy to determine the symmetries of a physical theory, namely, by determing those transformations of the variable space of the theory with respect to which the Lagrangian of the theory is invariant. ${ }^{2}$ See the appendix for a brief review of the symmetries of classical Yang-Mills gauge theories - the type of theory whose quantum version is the paradigm of a modern particle physics theory - as particularly relevant and illuminating examples.

If there are well-established procedures to determine a theory's symmetries, how can there still be a non-trivial challenge of clarifying which ontic variables designate identical physical states? As far as states of the entire universe (conceived of as a, or the, spatio-temporally maximal system) are concerned, there may indeed not be such a challenge: as forcefully argued by Baker (2011), there are good reasons to regard any pair of symmetry-related ontic variables that apply to the entire universe as designating one and the same physical state. For example, if one assumes that the laws of nature are rotation-invariant and considers the state of the entire universe rotated with respect to some fixed axis by some constant angle, it makes sense to regard the rotated state merely as a notational variant of the non-rotated state rather than as physically different. The assumption that symmetry-related universe states are physically identical is article.

However, with respect to subsystems of the universe- by which for the purposes of this paper I mean compact, finite proper subregions of the universe - it is not so clear whether one should really regard any two symmetry-related subsystem states as physically identical. To begin with, it is not entirely straightforward to define subsystem symmetries in the first place. The obvious definition is in terms of restrictions of universe symmetries to subsystems, but it is not immediately clear how to do this since universe symmetries may not leave the subsystems themselves

\footnotetext{
${ }^{2}$ The practical importance of this criterion notwithstanding the challenge of connecting empirical equivalence with any formal criterion for symmetries such as invariance of the Lagrangian under variable transformations is actually very complex. See (Belot 2011) for a study of "symmetry" and "physical equivalence" which explores and highlights some of the difficulties that arise from this challenge.

Note that in practice the heuristic challenge is typically opposite to the one depicted in the main text: rather than trying to determine the symmetries of an independently formulated theory from its Lagrangian, symmetries are imposed and one tries to construct a Lagrangian that exhibits them together with various other desiderata.
} 
invariant in the first place. For example, rotations of the Earth around its polar axis do not in general map continents onto themselves, even though continents appear to be obvious candidate subsystems of Earth's surface. The problem does not arise, however, for so-called internal symmetries, which leave space-time regions unaffected. Further technical challenges are raised by the fact that subsystems are in generally not physically isolated from their environment. This has the consequence that, by restricting a symmetry transformation to a subsystem of the universe, the resulting post-symmetry subsystem state may no longer give rise to a welldefined universe state when combined with the original environment state due to discontinuities on the subsystem boundary. ${ }^{3}$

Let us assume that we have managed to overcome these technical problems (e.g. by confining attention to symmetries that map subsystems onto themselves and considering appropriately isolated subsystems) and arrived at a conception of subsystem symmetries according to which indeed any symmetry-related subsystem ontic variables are empirically indistinguishable for observers inside the subsystem. One reason why one may still not want to regard all symmetry-related subsystem ontic variables as designating physically identical subsystem states is that symmetry-related subsystem ontic variables may well designate physical states that differ from the point of view of observers who are external to the subsystem. The present article explores the idea that two subsystem ontic variables designate one and the same physical subsystem state only if the states designated by them are empirically indistinguishable both from within the subsystem and from the point of view of arbitrary external observers. As it turns out, this idea can be connected in an illuminating way to a distinction among symmetries that philosophers have intensely discussed in the past few years, namely, that between symmetries which have direct empirical significance and symmetries which do not. In what follows I sketch the rationale that underlies this distinction.

As the philosophical debate about symmetries in physics has clarified ${ }^{4}$, symmetries can be empirically significant in various ways. Notably, their empirical significance is said to be direct if they relate subsystem states that, though empirically indistinguishable from the point of view of observers restricted to the subsystem, are empirically distinguishable from the perspective of external observers. Symmetries that do not have any direct empirical significance, in contrast, relate states that are empirically equivalent for all observers, whatever their position, and one may regard them as mere descriptive redundancies in that sense. Inasmuch as symmetries may empirically manifest themselves in other ways, e.g. by imposing constraints on the physical laws that connect empirical quantities, they are said to have indirect empirical significance. The most famous examples of such constraints are the conservation laws that follow from the Noether theorems.

Direct empirical significance is often illustrated in terms of Galileo's ship, a thought experiment that goes back to Galileo's Dialogue (Galileo 1953): as Salvatius highlights, there is no empirically detectable difference between what occurs in the cabin of a ship that is moving uniformly and what occurs in the cabin of a ship

\footnotetext{
${ }^{3}$ See (Greaves and Wallace 2014) for detailed reflections on this difficulty.

${ }^{4}$ See the anthology (Brading and Castellani 2003) for an overview of topics and relevant articles and (Brown and Sypel 1995, Kosso 2000, Brading and Brown 2004, Healey 2009, Greaves and Wallace 2014, Friederich 2015, Teh 2015) for contributions that are particularly relevant to the question of direct empirical significance. (Teh 2015, p. 99) provides a state-of-the-art side-by-side characterization of direct and indirect empirical significance.
} 
that is at rest with respect to the shore. The example can be used to highlight that due to the Galilean symmetries of the laws of motion, a boost of the ship to a constant velocity with respect to the shore is without any empirical consequences for observations confined to the cabin (which, absent stormy waters, is assumed to qualify as a sufficiently isolated subsystem). However, since boosting the ship to a constant velocity with respect to the shore creates an overall empirical difference with respect to external observers, e.g. observers watching from the shore, the boost transformation has direct empirical significance and is in that sense not a mere descriptive redundancy.

\section{Symmetries and physical identity}

Above we considered the idea of individuating subsystem physical states in terms of subsystem symmetries: to regard ontic variables $s$ and $s^{\prime}$ as designating one and the same physical subsystem state if they are symmetry-related, which, as we suppose, entails that they cannot be empirically distinguished from within the subsystem. The notion of direct empirical significance for symmetries suggests another, more fine-grained, way of individuating subsystem physical states: ontic variables $s$ and $s^{\prime}$ designate one and the same physical subsystem state if they are connected by a symmetry without any direct empirical significance. In other words, $s$ and $s^{\prime}$ designate the same physical state if they are empirically equivalent both from within the subsystem and from the perspectives of arbitrary external observers. In (Friederich 2015), I used this idea to spell out what it means for a subsystem symmetry to have direct empirical significance in terms of physical identity for subsystem states. Using the symbol " " to denote the relation of designating the same physical state between subsystem ontic variables, the criterion in question "DES" (which stands for "direct empirical significance") reads:

\section{Assumption (DES)}

A subsystem symmetry $\left.\sigma\right|_{S}$ has direct empirical significance if and only if $\left.\sigma\right|_{S}(s) \nsim s$ for some $s \in \mathcal{S}$,

where $\mathcal{S}$ is the state space associated with the subsystem $S$. In other words, according to DES, $\left.\sigma\right|_{S}$ does not have any direct empirical significance if and only if $\left.\sigma\right|_{S}(s) \sim s$ for all $s \in \mathcal{S}$.

Endorsing DES is not the only viable approach to make sense of the idea of direct empirical significance for subsystem symmetries. Another is to define physical identity only for universe states and to appeal to the constrained Hamiltonian formalism. (See (Earman 2003) for an eminent philosopher's enthusiastic endorsement of this formalism.) Symmetry transformations which, in this formalism's language, are generated by the so-called first-class constraints act trivially on observable quantities, so it makes sense to regard them as descriptive redundancies and identify them with the symmetries without direct empirical significance. Nicholas Teh (2015) has recently argued that according to this criterion there exist interesting analogies to the Galileo ship scenario in the gauge theories that underlie modern elementary particle physics. He takes his analysis to partly vindicates an earlier claim by Greaves and Wallace according to which some of the "local" gauge symmetries in gauge theories have direct empirical significance (Greaves and Wallace 2014), contrary to the claims made by other philosophers (Kosso 2000, Redhead 2002, Brading and Brown 2004, Lyre 2004, Healey 2009). Greaves and Wallace 
argue that the local gauge symmetries with direct empirical significance are those that do not reduce to the identity transformation on the subsystem boundary. Teh, however, shows that consistent application of the criterion that the symmetries without direct empirical significance are those that are generated by the first class constraints in the constrained Hamiltonian formalism yields a different result for some gauge theories, notably those in which some symmetry transformations connect topologically distinct field configurations (Teh 2015, sec. 4.2). ${ }^{5}$

As I shall argue in what follows, if - contrary to the approaches just mentioned that focus on the constrained Hamiltonian formalism - one accepts DES as constitutive of the connection between symmetries and the identity of physical states and adds two independently plausible assumptions concerning the identity of subsystem physical states, one comes to a conclusion about which symmetries have direct empirical significance that differs from those defended by Greaves and Wallace (2014) and Teh (2015). Namely, as I will show, one obtains a result according to which all (subsystem-restricted) gauge transformations in local gauge theories are without any direct empirical significance, whether or not they reduce to the identity transformation on the subsystem boundary and whether or not they connect topologically inequivalent configurations. Essentially the same result was already obtained in an earlier article (Friederich 2015), but the present article adds to the earlier considerations by showing that one of the assumptions used there - the assumption "Ext" - is not needed for the derivation of the result and by further motivating the other assumptions used there and here.

\section{Two assumptions}

The two announced assumptions concerning the identity of subsystem physical states can be formulated using a framework introduced by Greaves and Wallace (2014). The framework decomposes the universe $U$ into a subsystem $S$ and its mereological complement, the environment $E$, and it postulates associated state spaces $\mathcal{U}, \mathcal{S}$, and $\mathcal{E}$ for these systems. Elements $u \in \mathcal{U}$ are taken to be uniquely decomposable in terms of subsystem states $s \in \mathcal{S}$ and environment states $e \in \mathcal{E}$. The operation of combining a subsystem with an environment state is denoted by "*" (that is, $u=s * e$ is the universe state which arises from combining $s$ and $e$ ).

Arbitrary pairs of subsystem and environment states $s$ and $e$ need not in general give rise to a well-defined universe state $u=s * e$. For example, if $s$ and $e$ denote field configurations of a finite space-time region and its environment in a classical field theory, they do not in general coincide on the subsystem boundary, and in case they do, their derivatives may not coincide. In these cases, their composition need not be well-defined (depending on whether higher derivatives are required to exist etc.). However, in those cases where the composition $u=s * e$ of states $s \in \mathcal{S}$ and $e \in \mathcal{E}$ is well-defined, it is assumed to be unique.

Greaves and Wallace introduce subsystem and environment symmetries $\left.\sigma\right|_{S}$ and $\left.\sigma\right|_{E}$ as restrictions of universe symmetries $\sigma$ to the states spaces $\mathcal{S}$ and $\mathcal{E}$. They require that 'for all $s \in \mathcal{S}, e \in \mathcal{E}, \sigma(s * e)=\left.\left.\sigma\right|_{S}(s) * \sigma\right|_{E}(e)$ for some maps $\left.\sigma\right|_{S}$

\footnotetext{
${ }^{5}$ See (Strocchi 2015) for further reasons to accord special status to those symmetry transformations that are generated by the first class constraints, namely, that they entail a so-called local Gauss law, which imposes tight constraints on the empirical laws. In classical electromagnetism the local Gauss law corresponds to the Maxwell equations.
} 
and $\left.\sigma\right|_{E}$ [such that t] he symmetries $\Sigma_{S}$ of $\mathcal{S}$ and $\Sigma_{E}$ of $\mathcal{E}$ are just the sets of all such $\left.\sigma\right|_{S}$ and $\left.\sigma\right|_{E}$ respectively.' (Greaves and Wallace 2014, p. 68) They assume that restrictions $\left.\sigma\right|_{S}$ can be defined not only for internal symmetries $\sigma$ but also for space-time symmetries such as translations, boosts, and rotations. However, if $s * e$ is a well-defined universe state, the state $\left.\sigma\right|_{S}(s) * e$-where only $s$ but not $e$ has been subjected to the symmetry $\sigma$-need not be well-defined. In general, it is only well-defined if $\left.\sigma\right|_{S}$ is boundary-preserving on a class of states to which $s$ belongs. (Greaves and Wallace 2014, Sect. 5) Using this formal machinery, let us try to spell out what it might mean for two subsystem ontic variables to designate the same physical state in the spirit of the assumption DES.

By DES, symmetry-related subsystem states are physically different if the symmetry that connects them has direct empirical significance. For a symmetry to have direct empirical significance, in turn, the subsystem states that it connects must be, as Teh puts it, "empirically distinguishable with respect to a reference/environment system" (Teh 2015, p. 99, emphasis mine). Prima facie, a "reference system" need not be the same as an "environment system", and it may make sense to consider separately how one could empirically distinguish between subsystem states from the point of view of the environment (viewed as the rest of the universe) and from the point of view of another (finite and compact) proper subregion of the universe.

Let us first look at what it might mean for two subsystem states to be empirically distinguishable with respect to the environment. As an initial suggestion, one might use the idea that two subsystem states $s$ and $s^{\prime}$ are physically identical if an external observer, located in the subsystem's environment, could not distinguish between $s$ and $s^{\prime}$ by probing the subsystem using a measurement intervention. However, when taken literally, the idea leads to difficulties because probing a subsystem will in general affect its state and, by breaking the subsystem's isolation, may affect for which environment states $e \in \mathcal{E}$ both $s * e$ and $s^{\prime} * e$ are defined. An alternative implementation of what it might mean for two subsystem states to be empirically distinguishable with respect to the environment that is both straightforward and very general is the following: $s$ and $s^{\prime}$ are distinguishable from the point of view of some external observers if and only if, when combined with some suitable state of the environment $E$ in which an external observer may reside, they yield physically different universe states $s * e$ and $s^{\prime} * e$. Conversely, this means that the ontic variables $s$ and $s^{\prime}$ designate one and the same subsystem physical state if and only if, for arbitrary environment states $e \in \mathcal{E}$, the resulting universe states $s * e$ and $s^{\prime} * e$, if they are defined, are physically identical:

Assumption (SUL)

For all $s, s^{\prime} \in \mathcal{S}$ :

$s \sim s^{\prime}$, iff $s * e \sim s^{\prime} * e$ for all $e \in \mathcal{E}$ for which $s * e$ and $s^{\prime} * e$ are defined,

(where "SUL" stands for "subsystem-universe link").

We can illustrate the meaning of SUL by appeal to Galileo's ship (making the idealizing assumption that the ship is completely isolated with respect to the shore), where it yields the desired result that boosts do indeed have direct empirical significance: a situation $s^{\prime} * e$ in which the ship is moving ("boosted") with respect to the shore - which is part of the environment - is evidently physically different from a situation $s * e$ where it is at rest with respect to the shore, even though $s$ and $s^{\prime}=\left.\sigma\right|_{S}(s)$ (where $\left.\sigma\right|_{S}$ is the boost as applied to the ship alone) are empirically equivalent from inside the ship. SUL produces the result that the ship's unboosted 
and boosted states $s$ and $s^{\prime}$ are physically different, which, by DES, entails that the boost $\left.\sigma\right|_{S}$ has direct empirical significance, exactly as it should according to the discussion in section 2 .

Let us now look at what it might mean for two states $s_{1}$ and $s_{1}^{\prime}$ of a subsystem $S_{1}$ to be empirically distinguishable from the point of view of a second, external, reference subsystem $S_{2}$, which is now no longer the "environment" in the sense of the entire rest of the universe but a further compact and bounded proper subregion of the universe in its own right. As an example, $S_{1}$ could be Galileo's ship and $S_{2}$ could be the shore, considered as a separate subsystem rather than as part of the environment. Now consider the question whether if $s_{1}$ and $s_{1}^{\prime}$ are physically distinct states of Galileo's ship, they could still give rise to one and the same state of the joint subsystem "ship + shore" when combined with suitable "shore states" $s_{2}$ and $s_{2}^{\prime}$. In other words, could we "compensate" for a change in the state of the ship from $s_{1}$ to $s_{1}^{\prime}$ by adjusting the state of the shore from $s_{2}$ to $s_{2}^{\prime}$ such that the resulting physical state $s_{1}^{\prime} * s_{2}^{\prime}$ of the combined "ship + shore" system would be the same as the original state $s_{1} * s_{2}$ ?

Arguably, on an intuitively plausible reading of "physical state", the answer to this question is "no": to consider an example, assume that the ship states $s_{1}$ and $s_{1}^{\prime}$ are physically different in that the cabin colour differs between them. It would seem absurd to contemplate "balancing" this difference by adjusting the shore state between $s_{2}$ and $s_{2}^{\prime}$ such that the combined states $s_{1} * s_{2}$ and $s_{1}^{\prime} * s_{2}^{\prime}$ are physically identical. In other words, one cannot erase a physical difference such as that due to different cabin colours by adjusting the physical states of external subsystems such as the shore. Furthermore, even if $s_{1}$ and $s_{1}^{\prime}$, though physically different, are symmetry-related, e.g. an unboosted and a boosted state of the ship, it seems plausible that we cannot compensate for the difference between them, not even by "co-boosting" the shore from $s_{2}$ to $s_{2}^{\prime}$ : by DES and SUL, boosts of states of proper subsystems of the universe connect physically distinct subsystem states, and since the combined "ship + shore" subsystem is still a universe subsystem, the states $s_{1} * s_{2}$ and $s_{1}^{\prime} * s_{2}^{\prime}$ will still count as physically distinct. There is no motivation then to not consider $s_{1}$ and $s_{1}^{\prime}$ as physically distinct as well.

The considerations just developed are summed up in the following assumption:

Assumption (MAH)

For all $s_{1}, s_{1}^{\prime} \in \mathcal{S}_{1}$ and $s_{2}, s_{2}^{\prime} \in \mathcal{S}_{2}$,

if $s_{1} * s_{2} * e \sim s_{1}^{\prime} * s_{2}^{\prime} * e$ for all $e \in \mathcal{E}$ for which $s_{1} * s_{2} * e$ and $s_{1}^{\prime} * s_{2}^{\prime} * e$ are defined, then $s_{1} \sim s_{1}^{\prime}$ and $s_{2} \sim s_{2}^{\prime}$.

In (Friederich 2015), where this assumption was first introduced, it was characterized as a "modest anti-holism" - hence "MAH" - since it rejects the apparently holistic idea that one and the same physical state of affairs of a subsystem $S=S_{1} \cup S_{2}$ (where " $\cup$ " should be thought of as the mereological sum) can be "reduced" in various different ways, i.e. MAH rejects the "holistic" idea that the state of the whole can be decomposed in more than one way into physically distinct states of the parts. Another way to characterize MAH is as a weak mereological supervenience thesis: the complete physical state of the combined universe subsystem $S=S_{1} \cup S_{2}$ uniquely determines the physical states of its parts, the subsystems $S_{1}$ and $S_{2}$ (and, recursively, the physical states of the sub-subsystems of $S_{1}$ and $S_{2}$ ).

Note that MAH does not say that the states of the subsystem $S_{1}$ and $S_{2}$ jointly fix the state of the combined subsystem $S$, which would be a much less modest, 
indeed implausible, anti-holistic thesis. To appreciate the difference, assume for a moment that a quantum state (density matrix) $\rho$ were the true physical state of the subsystem $S=S_{1} \cup S_{2}$. Then one possible candidate for the physical state of the subsystem $S_{1}$ would be the reduced density matrix $\rho_{\text {red, }, 1}$ that is obtained from $\rho$ by "tracing out" the degrees of freedom associated with the second subsystem $S_{2}$. MAH would hold under these assumptions because $\rho_{\text {red, } 1}$ and $\rho_{\text {red,2 }}$ are uniquely determined by the state $\rho$ of the combined subsystem $S=S_{1} \cup S_{2}$ by the "tracing out" operation. However, famously, the reduced states $\rho_{\text {red,1 } 1}$ and $\rho_{\text {red }, 2}$ of the subsystems $S_{1}$ and $S_{2}$ do not uniquely determine the quantum state $\rho$ of the combined system, so the less modest anti-holistic thesis according to which the physical states of the subsystems $S_{1}$ and $S_{2}$ jointly fix the physical state of the combined subsystem $S$ fails in this case.

\section{Local gauge symmetries have no direct empirical significance}

In this section, I will re-derive a result concerning the direct empirical significance of so-called local gauge symmetries in gauge theories (Friederich 2015). Among these theories are the important Yang-Mills gauge theories, which are the backbone of contemporary elementary particle physics. (See the appendix for a brief review.) Without appealing to the additional assumption "Ext" used in (Friederich 2015) I show that, by DES, SUL, and MAH, the local gauge symmetries in these theories do not have any direct empirical significance. The decisive feature of local gauge symmetries that is needed to derive the result is that, when restricted to operating on a proper subgregion of the universe, they can always be extended to a symmetry operating on a more encompassing ("embracing") proper subregion in such a way that this extension reduces to the identity transformation on the boundary of the embracing region. The assumption "Ext" used in (Friederich 2015) postulates explicitly that the extended symmetry that operates on the embracing system be interior - i.e. that it have no direct empirical significance - but it turns out that this condition is itself derivable.

The announced result concerning the (lack of) direct empirical significance of gauge symmetries in local gauge theories is the following:

Proposition Given DES, SUL, and MAH, all local gauge symmetries in gauge theories (i.e. e.g. symmetries of the form (6) in Yang-Mills theories) do not have any direct empirical significance.

The derivation of this result goes as follows:

Let $S$ be a subsystem in the sense of the present formalism, i.e. a compact, finite proper subregion $S$ of the universe, and let $\left.\sigma\right|_{S}$ be some arbitrary local symmetry that is defined on the subsystem state space $\mathcal{S}$. Since $\left.\sigma\right|_{S}$ is a subsystem local gauge symmetry we can assume that, on an embracing subsystem $V \supset S$, the universe symmetry $\sigma$ of which $\left.\sigma\right|_{S}$ is a restriction can be restricted to a symmetry $\left.\sigma\right|_{V}$ of $\sigma$ which asymptotically approaches the identity transformation on the boundary of $V$, i.e. $\left.\sigma\right|_{V}$ is boundary-preserving on $V$. (In the appendix this is demonstrated in detail for the special case where the theory in question is a Yang-Mills gauge theory.) As a consequence, for any well-defined universe state $v * e$ with $v \in \mathcal{V}$ and $e \in \mathcal{E}$ (where $\mathcal{E}$ in this case is the state space associated with $E$, the mereological 
complement of $V$ ), the universe state $\left.\sigma\right|_{V}(v) * e$ is defined and physically identical with $v * e$, i.e.

$$
\left.v * e \sim \sigma\right|_{V}(v) * e .
$$

Using the assumption SUL we obtain that $\left.v \sim \sigma\right|_{V}(v)$. By DES, we conclude that $\left.\sigma\right|_{V}$ has no direct empirical significance.

Now, to show that $\left.\sigma\right|_{S}$ has no direct empirical significance either, consider the subsystem $S^{\prime}=V \backslash S$ (mereologically speaking, the part of $V$ that is not in $S$ ), and let $\mathcal{S}^{\prime}$ be the state space associated with $S^{\prime}$. Since $\left.\sigma\right|_{V}$ has no direct empirical significance, DES yields for arbitrary states $s \in \mathcal{S}$ and $s^{\prime} \in \mathcal{S}^{\prime}$ for which $s * s^{\prime}$ is defined:

$$
\sigma_{V}\left(s * s^{\prime}\right) \sim s * s^{\prime} .
$$

Using SUL, this gives for all $e \in \mathcal{E}$ for which $\sigma_{V}\left(s * s^{\prime}\right) * e$ and $s * s^{\prime} * e$ are defined:

$$
\sigma_{V}\left(s * s^{\prime}\right) * e \sim s * s^{\prime} * e .
$$

Decomposing $\left.\sigma\right|_{V}$ in terms of its restrictions $\left.\sigma\right|_{S}$ (the symmetry we were originally interested in) and $\sigma_{S^{\prime}}$ on the state spaces $\mathcal{S}$ and $\mathcal{S}^{\prime}$ we find that for all $e \in \mathcal{E}$ for which $\sigma_{V}\left(s * s^{\prime}\right) * e$ and $s * s^{\prime} * e$ are defined:

$$
\left.\sigma\right|_{S}(s) * \sigma_{S^{\prime}}\left(s^{\prime}\right) * e \sim s * s^{\prime} * e .
$$

Using MAH, it follows that

$$
\left.\sigma\right|_{S}(s) \sim s .
$$

Since $s$ was chosen arbitrary, Eq. (5) holds for all $s \in \mathcal{S}$. By DES, this establishes the proposition, namely, that $\left.\sigma\right|_{S}$ has no direct empirical significance.

Note that this result holds whether or not $\left.\sigma\right|_{S}$ itself is boundary preserving on $S$ (it suffices that it be extendable to some $\left.\sigma\right|_{V}$ that is boundary preserving on an embracing $V$ ). As highlighted in (Friederich 2015), this result shows that DES, SUL and MAH attribute direct empirical significance to fewer symmetries than the assumptions made by Greaves and Wallace (2014). Note, however, that the result does not carry over to global space-time symmetries, such as boosts, when restricted to subsystems. The later, as argued in the previous section, are identified as having direct empirical significance.

\section{Conclusion}

As remarked in Section 2, there are other ways to individuate subsystem physical states than through SUL and MAH. Furthermore, as remarked in Section 3, there are other ways to make the notion of direct empirical significance for symmetries precise than by appeal to DES, notably * by means of the constrained Hamiltonian formalism as done in (Teh 2015). There is no reason why one would have to make a definitive choice between these different approaches, i.e. there is no reason to oppose a healthy pluralism about ways to individuate subsystem physical states and about characterizations of what it means for symmetries to have direct empirical significance. 
To conclude in this pluralistic spirit, the main upshot of the present considerations is that (i) there are various viable accounts of physical identity for subsystem states, (ii) according to one viable account, encoded in SUL and MAH, subsystem physical states can be physically distinct even if they are empirically indistinguishable from the point of view of observers confined to the subsystem itself, and (iii) if one combines this account with an approach to the direct empirical significance of symmetries as encoded in DES, one obtains the result that the local gauge symmetries in gauge theories do not have any direct empirical significance.

\section{Appendix: Yang-Mills theories}

Yang-Mills theories are a generalization of classical relativistic electrodynamics, formulated in terms of so-called gauge field matrices $\mathbf{A}_{\mu}=\sum_{a} A_{\mu}^{a} T_{a}$, where the fields $A_{\mu}^{a}$ are analogous to the vector potential in electrodynamics and the $T_{a}$ are the so-called generators of the Lie algebra associated with the gauge group through which the theory is defined. In a proper Yang-Mills theory, this group is the noncommutative ("non-Abelian") $S U(N)$ group of all $N \times N$ unitary matrices with determinant 1 (for some integer $N$ ). In the Standard Model of elementary particle physics, the gauge groups $S U(2)$ and $S U(3)$ appear. One obtains electrodynamics if one takes the gauge group to be the (commutative) $U(1)$ group, whose only generator is the identity.

The Lagrangian of Yang-Mills theory is invariant with respect to local gauge symmetries, which are given by those transformation of the matrices $\mathbf{A}_{\mu}$ that are of the form

$$
\mathbf{A}_{\mu} \mapsto \mathbf{A}_{\mu}^{\prime}=\mathbf{U} \mathbf{A}_{\mu} \mathbf{U}^{\dagger}-\left(\frac{i \hbar}{g}\right)\left(\partial_{\mu} \mathbf{U}\right) \mathbf{U}^{\dagger}
$$

Here $g$ is the coupling constant that governs interactions in the theory (corresponding to the electric charge $e$ in electrodynamics). The space-time dependent unitary matrix $\mathbf{U}$ can be written in terms of some Hermitian matrix $\boldsymbol{\Lambda}$ as

$$
\mathbf{U}=\exp \left(-\frac{i g}{\hbar} \boldsymbol{\Lambda}\right)
$$

The entries of the matrix $\boldsymbol{\Lambda}$ are real-valued functions on space-time that parametrize all gauge transformations.

I will now show that, in this setting, any arbitrary local gauge symmetry $\left.\sigma\right|_{S}$ that is defined on some subsystem state space $\mathcal{S}$ can be extended to a local gauge symmetry $\left.\sigma\right|_{V}$ on the state space $\mathcal{V}$ associated with an embracing subsystem $V$ that is boundary preserving on $V$ :

Any subsystem local gauge symmetry $\left.\sigma\right|_{S}$ is associated with some Hermitian matrix $\boldsymbol{\Lambda}_{S}$ whose entries are real-valued functions on $S$. These functions can be smoothly extended beyond the boundary of $S$ on some embracing (still finite and compact) proper subregion $V \supset S$ of the universe to define a new Hermitian matrix $\Lambda_{V}$ whose entries are functions that tend smoothly towards zero in a neighbourhood of the boundary of $V$. The newly obtained matrix $\boldsymbol{\Lambda}_{V}$, in turn, defines a symmetry $\left.\sigma\right|_{V}$ which acts on the elements of $\mathcal{V}$, the state space associated with $V$. Since the entries of $\Lambda_{V}$ are zero in a neighbourhood of the boundary of $V$, the symmetry $\left.\sigma\right|_{V}$ is boundary presering on $V$, and the transformation $\left.\sigma\right|_{V} * \mathbf{1}_{E}$ is defined and a universe symmetry. This, furthermore, entails that, for any well-defined universe 
state $v * e$ with $v \in \mathcal{V}$ and $e \in \mathcal{E}$, the universe state $\left.\sigma\right|_{V}(v) * e$ is also defined and physically identical with $v * e$.

\section{References}

Baker, David John. 2011. Broken symmetry and spacetime. Philosophy of Science 78: $128-48$.

Belot, Gordon. 2011. Symmetry and equivalence. In Philosophy of Science Assoc. 22nd Biennial Mtg. (Montréal, QC) > PSA 2010 Contributed Papers.

Brading, Katherine and Harvey R. Brown. 2004. Are gauge symmetry transformations observable?. British Journal for the Philosophy of Science 55: 645-65.

Brading, Katherine and Elena Castellani (eds.). 2003. Symmetries in physics: Philosophical reflections. Cambridge: Cambridge University Press.

Brown, Harvey R. and Roland Sypel. 1995. On the meaning of the relativity principle and other symmetries. International Studies in the Philosophy of Science 9: $235-53$

Earman, John. 2003. Tracking down gauge: An ode to the constrained Hamiltonian formalism. In ((Brading and Castellani 2003)), 140-162.

Friederich, Simon. 2015. Symmetry, empirical equivalence, and identity. British Journal for the Philosophy of Science 66: 537-559.

Galileo. 1953. Dialogue concerning the two chief world systems, Berkeley: University of California Press.

Greaves, Hilary and David Wallace. 2014. Empirical consequences of symmetries. British Journal for the Philosophy of Science 65: 59-89.

Healey, Richard. 2009. Perfect symmetries. British Journal for the Philosophy of Science 60: 697-720.

Leifer, Matthew Saul 2014. Is the quantum state real? An extended review of $\psi$-ontology theorems. Quanta 3: 67-155.

Lyre, Holger. 2004. Holism and structuralism in U(1) gauge theory. Studies in History and Philosophy of Modern Physics 35: 643-70.

Kosso, Peter. 2000. The empirical status of symmetries in physics. British Journal for the Philosophy of Science 51: 81-98.

Redhead, Michael L. G. 2002. The interpretation of gauge symmetry. In Ontological aspects of quantum field theory, ed. Meinard Kuhlmann, Holger Lyre, and Andrew Wayne. 303-312. Singapore: World Scientific.

Strocchi, Franco. 2015. Symmetries, symmetry breaking, gauge symmetries. http://arxiv.org/abs/1502.06540, accessed 26 February 2016.

Teh, Nicholas Joshua. 2015. Galileo's gauge: Understanding the empirical significance of gauge symmetry. Philosophy of Science 83: 93-118. 